\title{
THE QUANTUM METROLOGY TRIANGLE EXPERIMENT: QUANTIZATION TESTS OF AN ELECTRON PUMP
}

\author{
S. Sassine*, B. Steck*, N. Feltin*, L. Devoille*, B. Chenaud*, W. Poirier*, \\ F. Schopfer*, G. Spengler*, O. Séron*, F. Piquemal*, S. Lotkhov ${ }^{\dagger}$
}

*Laboratoire National de Métrologie et d'Essais (LNE),

29 avenue Roger Hennequin, F-78197 Trappes, France

†Physikalisch-Technische Bundesanstalt (PTB),

Bundesallee 100, D-38116 Braunschweig, Germany

\begin{abstract}
We present our experimental set-up and discuss the results obtained with the quantum metrological triangle (QMT) experiment. This experiment consists in realizing Ohm's law with the three effects used and investigated in quantum electrical metrology: the Josephson effect (JE), the quantum Hall effect (QHE) and the single electron tunneling effect (SET). The aim is to check the consistency of the phenomenological constants $K_{J}, R_{K}$ and $Q_{X}$ associated with these effects and theoretically expressed with the fundamental constants $e$ and $h$ (elementary charge and Planck constant, respectively). Such an experiment is a contribution for a new definition of electrical units in the International System (SI).
\end{abstract}

KEYWORDS: electrical metrology, low current measurements, cryogenic current comparator, quantum metrological triangle, quantum current standard.

\section{INTRODUCTION}

More than twenty years ago, profound changes occurred in electrical metrology with the introduction of quantum physics. The quantum effects enabled the development of standards that are more stable, more robust, more repro-

Artigo submetido em 12/10/2009 (Id.: 01057)

Revisado em 27/03/2010, 22/10/2010

Aceito sob recomendação do Editor Associado Prof. Sebastian Yuri Cavalcanti Catunda ducible and more accurate. Since this time, the reference standards of resistance and electrical voltage are based on quantum Hall (QHE) and Josephson effects (JE), which are linked to the von Klitzing constant $R_{K}$ and the Josephson constant $K_{J}$, respectively. These quantum effects insure for the corresponding units, the ohm and the volt, a high level of reproducibility (as high as a few parts in $10^{11}$ for the JE and a few parts in $10^{9}$ for the QHE) and a unique representation all over the world. Thus nowadays, since an accurate current standard is not available, the ampere is experimentally produced by means of the practical volt and ohm originating from the JE and QHE, respectively, via the Ohm's law.

At the same time, the advent of the single-electron devices can pave the way for developing a new current standard with an accuracy better than that obtained with the material standards up to now (Zimmerman and Keller 2003; Graber and Devoret 1992). The principle is based on a phenomenon called single-electron tunneling (SET) which ensures a quantized flow of charges through a nano-device. For 15 years, the development of the nanofabrication has made possible the realization of these quantum devices enabling the manipulation of individual electrons.

In the framework of the redefinition of the SI base units in term of a reduced set of fundamental constants, the quantum metrological triangle is a key experiment (Likharev and Zorin 1985; Keller 2008)since the challenge is to redefine the whole electrical units from only two constants ( $h$ and $e$ ). 
The basic principle is represented in figure 1 and consists in applying a "quantum” Ohm's law (Piquemal and Genevès 2000):

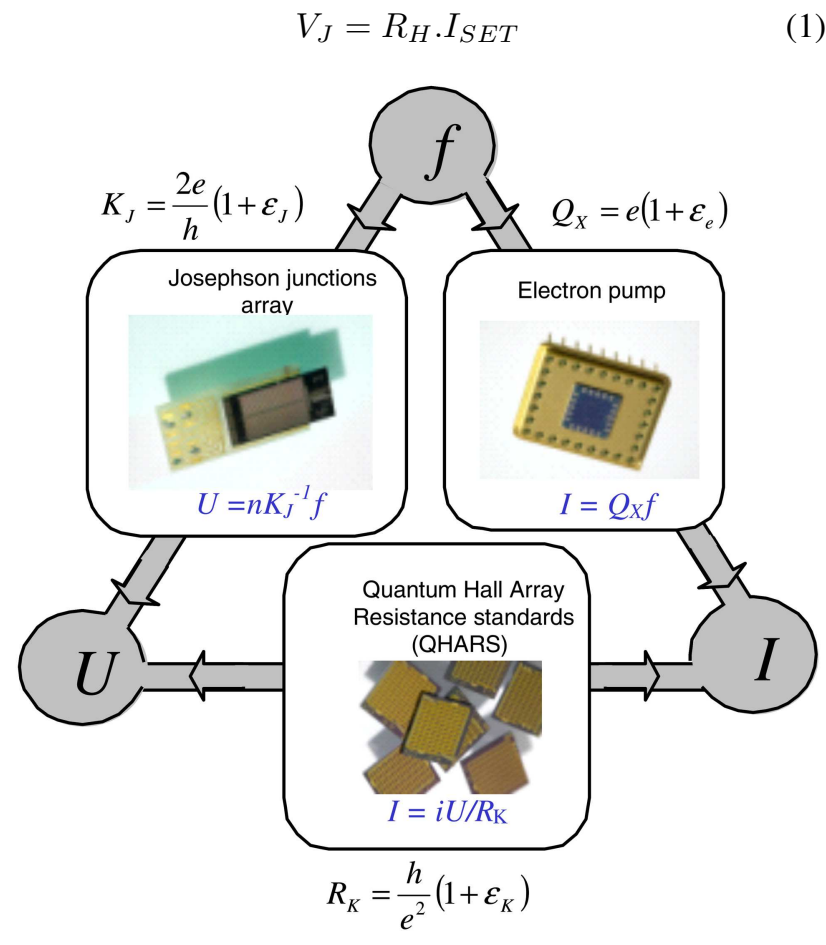

Figure 1: Principle of the QMT. $\epsilon_{J}, \epsilon_{K}$ and $\epsilon_{e}$ represent the correction factors on $K_{J}, R_{K}, Q_{X}$.

$V_{J}$ and $R_{H}$ denote the Josephson voltage and the Hall resistance respectively. $I_{S E T}$ corresponds to a current generated by a SET device. This is the direct way for closing the QMT.

Another method is the indirect way which consists in developing a quantum capacitance standard from SET devices, called Electron Counting Capacitance Standard (ECCS). This experiment firstly implemented by the NIST allowed to successfully closing the QMT with a relative standard uncertainty of 9.2 parts in $10^{7}$ (Keller, Zimmerman and Eichenberger 2007). The principle is simply based on the natural definition of capacitance. The experimental set-up is completely described in (Keller, Zimmerman and Eichenberger 2007).

In our experiment, the SET devices are metallic 3-junctions single electron pumps in aluminium consisted of two gate electrodes and on-chip resistors at the ends in order to reduce the co-tunneling effect (Lotkhov et al. 2001; Steck et al. 2008) . The quantized current generated by this pump is theoretically equal to $e . f_{S E T}$ ( $f_{S E T}$ denotes the pumping frequency applied to the gates) and is measured through a cryogenic current comparator (CCC), which allows to amplify the low SET current with a metrological accuracy.

Practically, the closure of the QMT is a measurement of the product $R_{K} K_{J} Q_{X}$, theoretically equal to $2\left(K_{J}=2 e / h\right.$ is the Josephson constant, $R_{K}=h / e^{2}$ the von Klitzing constant and $Q_{X}=e$ an estimate of the electron charge). Checking the equality $R_{K} K_{J} Q_{X}=2$ at an uncertainty level of 1 part in $10^{8}$ is the ultimate aim of this experiment and will be a significant contribution to the redefinition of electrical units from $(h, e)$.

Moreover, the development of a SET current standard could lead to the definition of a quantum ampere as the product of the elementary charge and a frequency. The classical definition of the ampere is: "The ampere is that constant current which, if maintained in two straight parallel conductors of infinite length, of negligible circular cross-section, and placed 1 metre apart in vacuum, would produce between these conductors a force equal to $2 \times 10^{-7}$ newton per metre of length". This definition fixing $\mu_{0}$ (vacuum permeability) allows relating the electrical units to the mechanical units via a force, but the experimental realization of the current unit called "ampere balance" is not used any more. In this experiment, a current flew through two coils and the interaction force between these two coils was measured. The main limitation of this standard was due to the imperfect determination of the geometry of the coils, so the ultimate achieved uncertainty was $5 \times 10^{-6}$ (Elmquist et al. 2001).

In the context of the current discussion about revising the SI based on fundamental constants, the use of a quantum ampere standard could be the most suitable way for the "mise en pratique" of the ampere if it is redefined as: "The ampere is the electrical current equivalent to the flow of exactly $1 /\left(1.602176487 \times 10^{-19}\right)$ elementary charges per second" (Mills et al. 2006) . This definition fixes the elementary charge as exactly $1.602176487 \times 10^{-19} \mathrm{~A} \cdot \mathrm{s}$. The value of $e$ taken here is the 2006 CODATA recommended value.

\section{EXPERIMENTAL DESCRIPTION}

Our implementation of the direct closure of the QMT is shown in figure 2: the current supplied by the SET source is amplified, then feeds a resistor $R_{\text {cal }}$ calibrated against $R_{K}$ by means of the QHE and the voltage induced at the terminals of the resistor is compared, in real time, with the voltage $V_{J}=n_{J} f_{J} / K_{J}$ generated by a Josephson array voltage standard (using $n_{J}$ junctions irradiated by an RF electromagnetic wave with the frequency $f_{J}$ ). In fact, for practical reasons, the QHE is not directly involved in our experiment. Instead, a material resistance calibrated by means of the QHE is used and located at room temperature outside the dilution unit (see figure 2). The maximum frequency at which the SET device can be driven is limited to $100 \mathrm{MHz}$, which corresponds to a quantized current of around $16 \mathrm{pA}$. This very weak current is amplified and measured through the CCC. The CCC is a metrological tool with high performance initially developed 


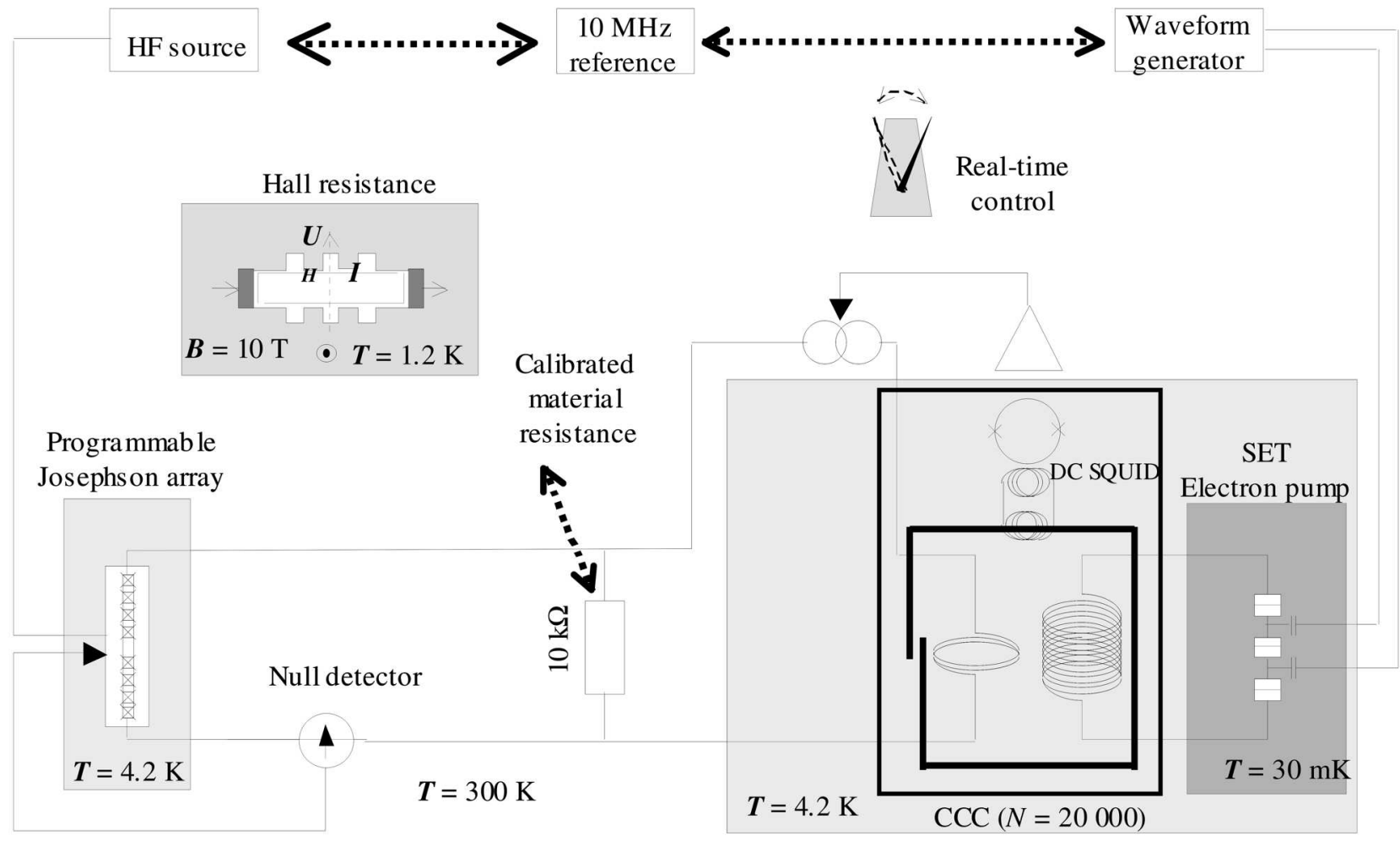

Figure 2: Our experimental set-up for the direct closure of QMT.

for the very accurate comparison of resistances. It can be also used as an amplifier with a very accurate known gain (better than $10^{-8}$ ) (Gay,Piquemal and Genevès 2000; Gallop and Piquemal 2006). It also has an excellent current resolution (around $1 \mathrm{fA} / \mathrm{Hz}^{1 / 2}$ in the white noise regime). The QMT's CCC consists of two windings of $N_{1}=20000$ turns and $N_{2}=1$ turn embedded in a superconducting toroïdal shield associated with a magnetic flux detector which is a DC SQUID (Superconducting Quantum Interference Device), and its gain is exactly equal to the winding ratio ( $G=$ $N_{1} / N_{2}$ ). The first winding is directly connected to the pump and is flowed by the quantized current $I_{S E T}$ while the second winding is fed by a feedback current $I_{F B}=G \cdot I_{S E T}$ supplied by a home-made external stable current source. The synchronization between the Josephson array and the current supplied by the SET device is ensured by Digital Analogical Converters (DAC) driven by a computer (not represented in figure 2). The working principle of the home-made current source is the following : the voltage signal coming from a DAC, which supplies the main value of the feedback signal (adjusted to a value close to 20000. $I_{S E T}$ ), and the feedback signal of the Squid electronics (the small deviation measured by the Squid), are isolated seperately through high input impedance differential amplifiers. Then these voltage signals are summed and then converted to current through a voltage-current conversion circuit having a current range be- tween $20 \mathrm{nA}$ and $1 \mu \mathrm{A}$. A scheme of this home-made current source is presented in figure 3 .

In practice, the relation (1) becomes:

$$
V_{J}=R_{c a l} \cdot G \cdot I_{S E T}
$$

Preliminary results obtained with the complete QMT set-up have demonstrated the feasibility of the experiment. The null detector (which is an Agilent 3458A 81/2 Digit Multimeter) shown in figure 2 measures the difference between the Josephson voltage and the voltage drop at the terminals of the calibrated resistor, $V_{d}=V_{J^{-}} V_{R}$, with $V_{R}=R_{c a l} . I_{F B}$. From this datum and for testing the measurement bench, the quantity $\Delta e / e$ has been determined in the following way:

where

$$
\frac{\Delta e}{e}=\frac{Q_{x}-e_{C O D A T A}}{e_{C O D A T A}}
$$

$$
Q_{x}=\left(\frac{1}{G \cdot f_{S E T} \cdot R_{c a l}}\right) \cdot\left(\frac{n_{J} f_{J}}{K_{J}}-V_{d}\right)
$$

$\Delta e / e$ corresponds to the discrepancy between the CODATA value of the elementary charge (Mohr, Taylor and Newell 2008) and the single charge $Q_{X}$ of our SET pump. In other words, this measurement allows to estimate the exactness of the SET device or the deviation from the current quantization $\left(e . f_{S E T}\right)$. 


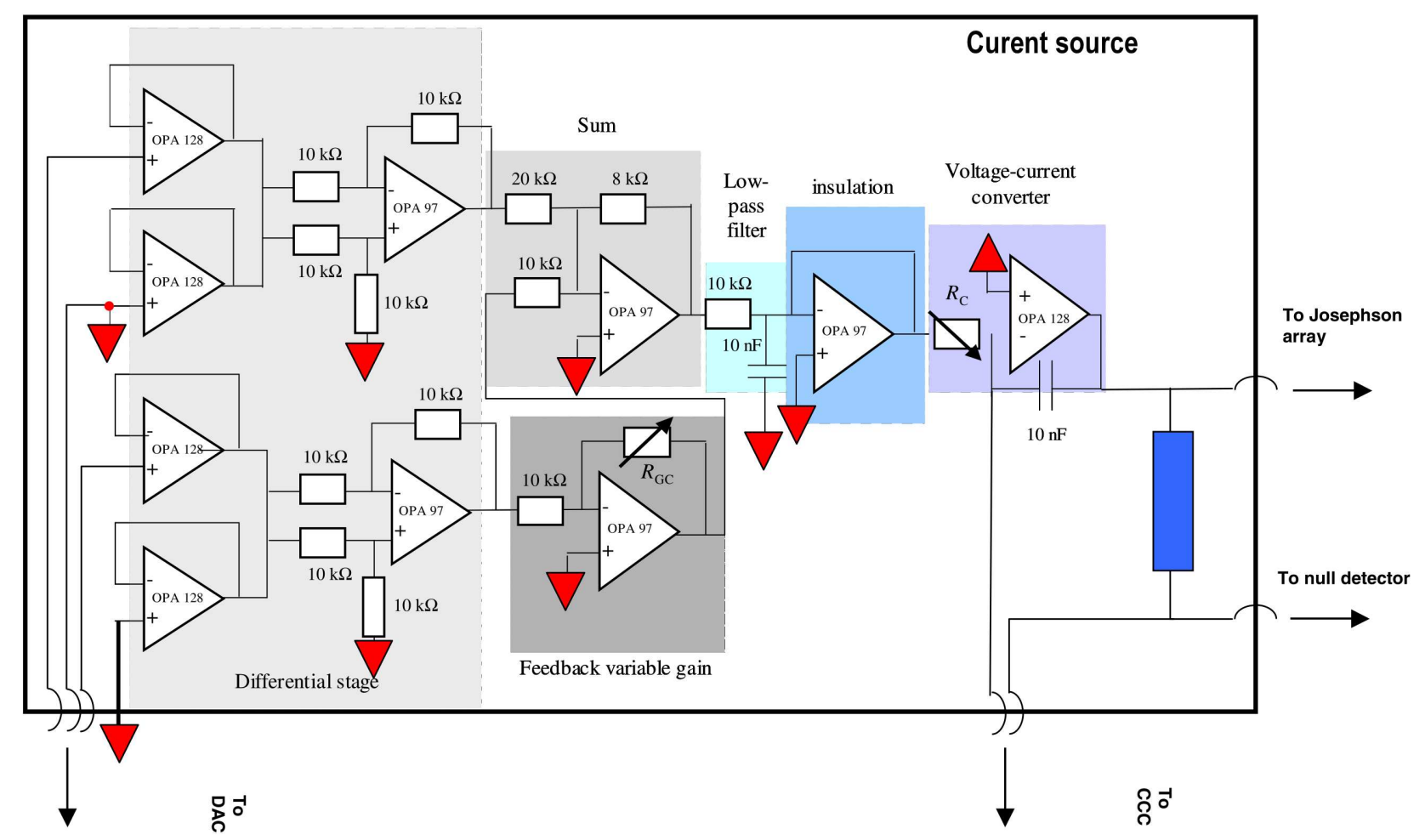

Figure 3: Scheme of the home-made current source.

\section{RESULTS}

The various parameters of the SET pump have been estimated from the experimental stability diagram as described in a previous paper (Steck et al. 2008) . The three junction capacitances $C_{L}, C_{m}, C_{R}$ (left, middle and right resp.) have been found to be $C_{L}=115 \mathrm{aF}, C_{m}=80 \mathrm{aF}, C_{R}=$ $115 \mathrm{aF}$. The two gate electrodes controlling the charging states of the islands have capacitances of $C_{g 1}=35 \mathrm{aF}, C_{g 2}$ $=30 \mathrm{aF}$. The parameters characterizing the cross-talking effect due to the proximity of islands, defined in (Steck et al. 2008), have been found to be $f_{1}=0.3$ and $f_{2}=0.25$. A white noise level close to $3 \mathrm{fA} / \mathrm{Hz}^{1 / 2}$ has been measured with the complete measuring system at $1 \mathrm{~Hz}$. A large series of current plateau measurements $\left(\mathrm{I}_{\text {pump }} / \mathrm{V}_{\text {bias }}\right)$ has been performed with the complete experimental set-up (figure 2) in the frequency range $10-100 \mathrm{MHz}$ by measuring the voltage at the $R_{c a l}$ terminals by means of a multimeter (figure 4). Negative current steps have been carried out by phase shifting the second harmonic signal (Steck et al. 2008) .

As expected, when the frequency is increased the plateau width decreases and the centre of the plateau where the cotunnelling is minimized shifts towards larger bias voltage.

As shown in figure 5 (blow-up of two current steps for 10 $\mathrm{MHz}$ and $30 \mathrm{MHz}$ pumping frequencies) the current plateau

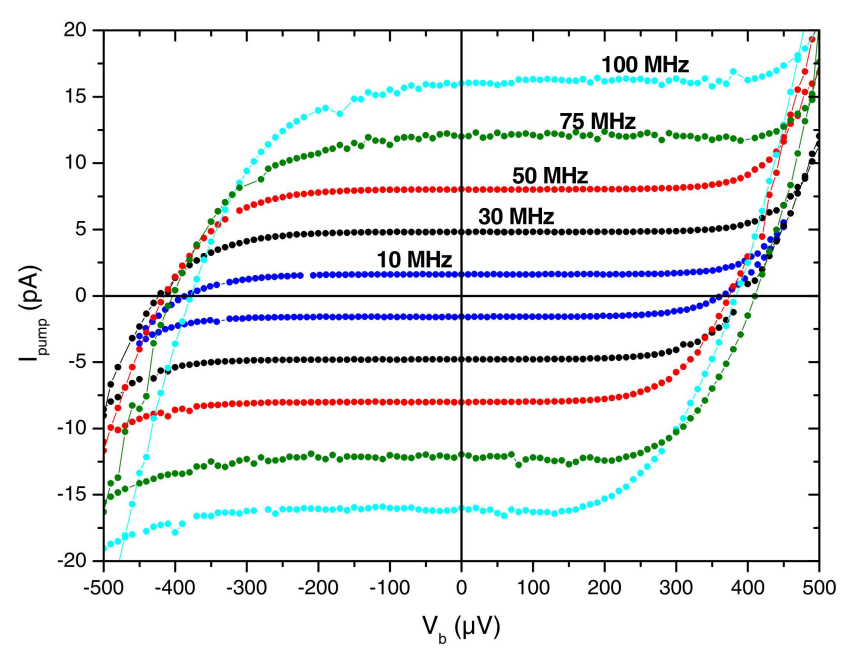

Figure 4: A series of current plateaus in the frequency range 10-100 $\mathrm{MHz}$.

is flat over a relatively large bias voltage range, $350 \mu \mathrm{V}$ for 30 $\mathrm{MHz}$ and $390 \mu \mathrm{V}$ for $10 \mathrm{MHz}$, within the noise floor $\Delta I_{n f}$ $=40 \mathrm{fA}$. These results are similar to those obtained in internal feedback mode where the output current of the SQUID electronics is fedback directly to the SQUID and not in the secondary winding of the CCC (Steck et al. 2008).

Then, long-term measurements have been performed in order 


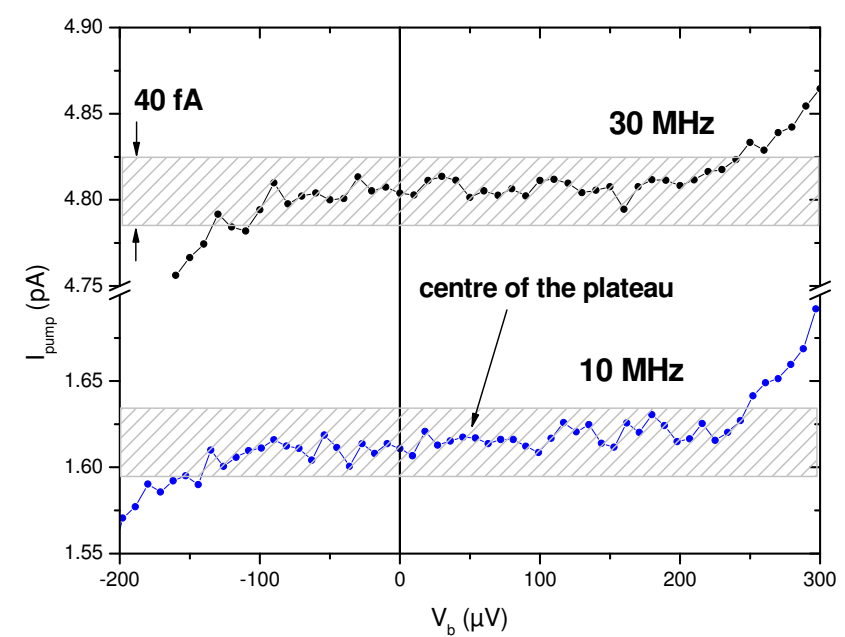

Figure 5: Blow-up of two current plateaus obtained at $10 \mathrm{MHz}$ and $30 \mathrm{MHz}$ pumping frequencies. The hatched parts represent the $40 \mathrm{fA}$ noise for a 5 minutes measuring time.

to reduce random uncertainty. The bias voltage is adjusted in such a way that the generated current corresponds to the center of the plateau. Investigations about the time domain stability of a R-pump have demonstrated that $1 / f$ noise occurs very early after roughly $100 \mathrm{~s}$ (Steck et al. 2008). The appearance of a $1 / f$ regime displays high correlations among the measurable quantities and limits the reduction of the random uncertainty. The $1 / f$ noise can be avoided by periodically reversing the SET current with modifying, for instance, the phase shift between the two gate harmonic signals.

As a consequence, the complete system including the stable current source and the DC SQUID has to be especially designed for quick (roughly at the rate of $1 \mathrm{~Hz}$ ) periodic inversions of the current. The synchronization between the Josephson array and the current supplied by the SET device is ensured by Digital Analogical Converters (DAC) driven by a computer (not represented in the figure 2).

From measurements carried out over measuring time as long as 10 hours, Allan standard deviations have been calculated for two different frequencies and reported in figure 6. Five (eight, resp.) junctions of the Josephson array have been biased for balancing the potential drop induced by the current of the pump driven at $23.55 \mathrm{MHz}$ (37.69 MHz, resp.).

The Allan standard deviation, firstly developed by the time and frequency community, is used for describing the noise nature over long time ranges and for revealing the possible correlations among data. Moreover, it is a convenient statistical tool because in white noise regime, i.e. when the data are uncorrelated, the Allan variance and the experimental variance of the mean are unbiased estimates of the theoretical variance. As a consequence, the experimental standard deviation of the mean (ESDM), recommended in the GUM for

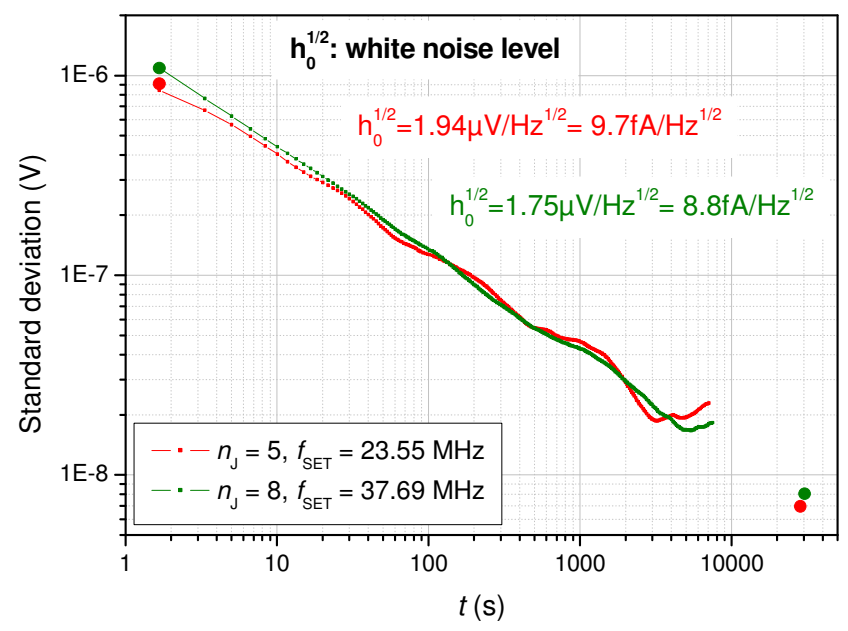

Figure 6: Allan standard deviations (dots and lines) and experimental standard deviation of the mean (large points) calculated for two different frequencies $f_{S E T}=23.55 \mathrm{MHz}$ and $37.69 \mathrm{MHz}$ corresponding to 5 and 8 biased junctions of the Josephson array respectively.

characterizing the type A uncertainty, can directly be read on the Allan deviation curve shown in figure 6 since this curve varies according to a $\tau^{-1 / 2}$-law distinguishing a white noise regime.

From measurements of $V_{d}$ in readout of the null detector, the quantity $\Delta e / e$, discrepancy between $Q_{X}$ and the CODATA recommended value of $e$ (Lotkhov et al. 2001) has been calculated and reported in figure 7. For each value, the ESDM calculated through Allan deviation is given. Within one run corresponding to 6 measurements (figure 7) and with a given adjustment of the parameters, a consistent set of data has allowed to achieve a weighted random uncertainty of 4 parts in $10^{6}$. A similar result ( 7 parts in $10^{6}$ ) has been obtained with a second pumping frequency $\left(f_{S E T}=23.55 \mathrm{MHz}\right)$ and $5 \mathrm{bi}-$ ased Josephson junctions. However, discrepancies as large as 1.5 parts in $10^{4}$ have been measured on the absolute value of SET current from a run to the other (figure 8). Some changes on the experimental set-up (shielding, ground...) are in progress in order to suppress these irreproductibilities.

\section{CONCLUSION}

These preliminary results prove that the direct QMT set-up never implemented up to now is operating. The final random uncertainty of $4 \mathrm{ppm}$ is encouraging to hope to close the QMT at a $1 \mathrm{ppm}$ level considered as a first important step in the near future. First of all, the troubles related to the non reproducible measurements must be solved. But we can already claim that the QMT set-up is the best measuring bench for testing the exactness of the current generated by the single electron devices. A new generation of SET devices able to generate currents as high as $1 \mathrm{nA}$ is expected for replacing 


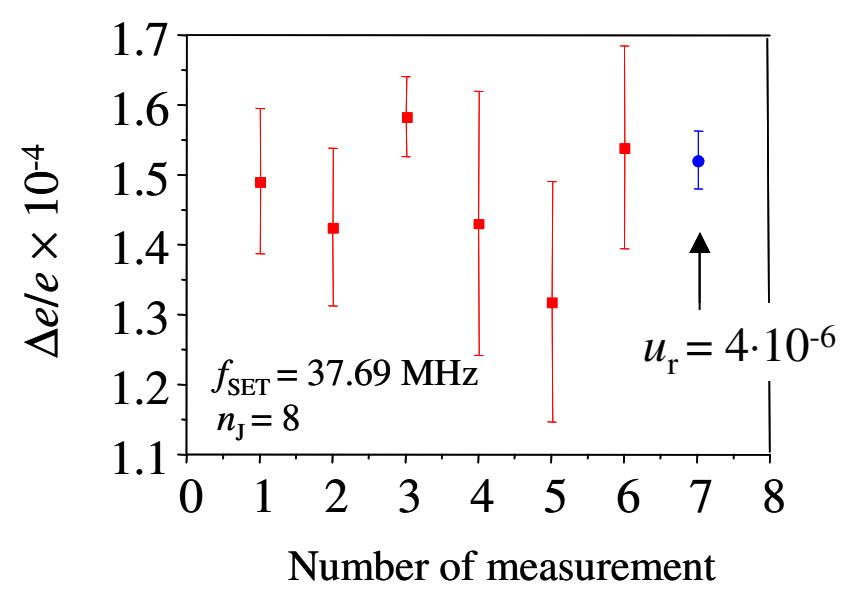

Figure 7: A series of 6 measurements performed at a $37.69 \mathrm{MHz}$ pumping frequency over a run. The blue point is the weighted mean associated with its relative uncertainty $u_{r}$.

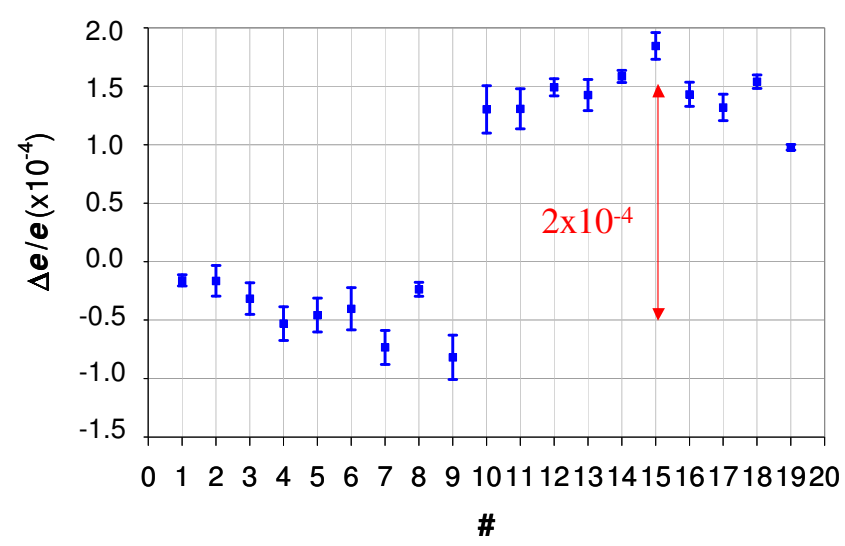

Figure 8: Discrepancies observed from one run to another for $f_{S E T}$ $=37.69 \mathrm{MHz}$ and 8 biased junctions.

the aluminium devices (Fujiwara, Nishiguchi and Ono 2008; Blumenthal et al. 2007). Consequently the set-up described in this work will be able to be used to verify the quantization of the current they supply.

Afterwards, checking the equality $R_{K} K_{J} Q_{X}=2$ with lower uncertainties for preparing the new S.I. induces the use of a new type of CCC and/or implementation of a new SET candidate. For instance, CCCs consisted of more sensitive SQUID are presently developed in our laboratory.

Finally, in case that the $10^{-8}$ targeted uncertainty was achieved in the QMT and the watt balance experiments, then the set of experiments \{QMT, watt balance, calculable capacitor (in which the $10^{-8}$ uncertainty is already achieved) could be an alternative for replacing the calculation given by the relation $e=\left[2 \alpha h /\left(\mu_{0} c\right)\right]^{1 / 2}$ in the CODATA adjustment by a direct determination of $e[8,16]$.

In summary, in the framework of a modification of the SI, the closure of the QMT will have major implications for electrical units. Indeed, a successful outcome both for the Watt balance and for the QMT would make it possible to link the kilogram to $h$ and the Ampere to $e$. In this case, a determination of $e$ and a sufficient confidence in the relations $K_{J}=$ $2 e / h$ and $R_{K}=h / e^{2}$ are needed. As shown above, the QMT can strengthen this confidence and allows a determination of the charge quantum $Q_{X}$ in the SI of units.

\section{AKNOWLEDGMENTS}

This work was partially funded by the French National Research Agency project n ${ }^{\circ}$ ANR-05-JCJC-0089-01 and by the European Community's Seventh Framework Program "ERA-NET PLUS" (Grant Agreement N²17257) within the EURAMET Joint Research Project "REUNIAM".

\section{REFERENCES}

Blumenthal, M.D. et al. (2007). Gigahertzquantized charge pumping. Nature Physics, 3, pp. 343-347.

Elmquist, R.E. et al. (2001). The ampere and electricalstandards. Journal of Research of the National Institute ofStandards and Technology, 106, pp. 65-103.

Fujiwara, A., K. Nishiguchi, and Y. Ono (2008).Nanoampere charge pump by single-electron ratchet using siliconnanowire metal-oxide-semiconductor field-effect transistor.Applied Physics Letters, 92:4, pp. 042102.

Gallop, J. and F. Piquemal (2006). in The SQUIDHandbook, Applications of SQUIDs and SQUID Systems. Vol. II, Ch. 9, p. 95, Eds. J. Clarke and A. Braginski. Weinheim,Wiley-VCH.

Gay, F., F. Piquemal, and G. Genevès (2000). Ultralow noise amplifier based on a cryogenic current comparator.Review of Scientific Instruments, 71:12, pp. 45924595 .

Graber, H. and M.H. Devoret (1992). Single ChargeTunneling. NATO Advanced Studies Institute, Series B: Physics, Vol.294. Plenum, New York.

Keller, M.W., A.L. Eichenberger, J.M. Martinis, and N.M. Zimmerman(1999). A capacitance standard based on counting clectrons.Science, 285, pp. 1706-1709.

Keller, M.W., F. Piquemal, N. Feltin, B. Steck, and L.Devoille (2008). Metrology triangle using a Watt balance, a calculablecapacitor and a single-electron tunneling device. Metrologia,45:3, pp. 330-334.

Keller, M.W., N.M. Zimmerman, and A.L. Eichenberger(2007). Uncertainty Budget for the NIST Elec- 
tron Counting CapacitanceStandard, ECCS-1. Metrologia, 44:6, pp. 505-512.

Likharev, K.K. and A.B. Zorin (1985). Theory of theBlochwave oscillations in small Josephson junctions. Journalof Low Temperature Physics, 59, pp. 347-382.

Lotkhov, S.V., S.A. Bogoslovsky, A.B. Zorin, and J.Niemeyer (2001). Operation of a three-junction single-electron pumpwith on-chip resistors. Applied Physics Letters, 78:7, pp.946-948.

Mills, I.M., P.J. Mohr, T.J. Quinn, B.N. Taylor, and E.R. Williams(2006). Redefinition of the kilogram, ampere, kelvin and mole: aproposed approach to implementing CIPM Recommendation 1 (CI-2005).Metrologia, 43:3, pp. 227-246.

Mohr, P.J., B.N. Taylor, and D.B. Newell (2008). CODATArecommended values of the fundamental physical constants: 2006.Review of Modern Physics, 80:2, pp. 633-730.

Piquemal, F. and G. Genevès (2000). Argument for adirect realization of the quantum metrological triangle.Metrologia, 37:3, pp. 207-211.

Steck, B. et al. (2008). Characterization andmetrological investigation of an R-pump with driving frequencies up to100 MHz. Metrologia, 45:4, pp. 482-491.

Zimmerman, N.M. and M.K. Keller (2003). Electrical metrology withsingle electrons. Measurement Science and Technology, 14, pp.1237-1242. 